\title{
Improved Electrical Conductivity of Carbon/Polyvinyl Alcohol Electrospun Nanofibers
}

\author{
Nader Shehata, ${ }^{1,2}$ Nabil Madi, ${ }^{3}$ Mariam Al-Maadeed, ${ }^{3}$ \\ Ibrahim Hassounah, ${ }^{4}$ and Abdullah Ashraf ${ }^{3}$ \\ ${ }^{1}$ Department of Engineering Mathematics and Physics, Faculty of Engineering, Alexandria University, Elhadara, \\ Alexandria 21544, Egypt \\ ${ }^{2}$ Center of Smart Nanotechnology and Photonics (CSNP), SmartCI Research Center, Alexandria University, Elhadara, \\ Alexandria 21544, Egypt \\ ${ }^{3}$ Center of Advanced Materials (CAM), Qatar University, Doha 2713, Qatar \\ ${ }^{4}$ USTAR Bioinnovation Center, Utah State University, Logan, UT 84341, USA \\ Correspondence should be addressed to Nader Shehata; nader83@vt.edu
}

Received 6 June 2015; Revised 24 July 2015; Accepted 3 August 2015

Academic Editor: Mircea Chipara

Copyright (C) 2015 Nader Shehata et al. This is an open access article distributed under the Creative Commons Attribution License, which permits unrestricted use, distribution, and reproduction in any medium, provided the original work is properly cited.

\begin{abstract}
Carbon nanofibers (CNFs) gained much interest in the last few years due to their promising electrical, chemical, and mechanical characteristics. This paper investigates a new nanocomposite composed of carbon nanofibers hosted by PVA and both are integrated in one electrospun nanofibers web. This technique shows a simple and cheap way to offer a host for CNFs using traditional deposition techniques. The results show that electrical conductivity of the formed nanofibers has been improved up to $1.63 \times$ $10^{-4} \mathrm{~S} / \mathrm{cm}$ for CNFs of weight $2 \%$. The peak temperature of mass loss through TGA measurements has been reduced by $2.3 \%$. SEM images show the homogeneity of the formed PVA and carbon nanofibers in one web, with stretched CNFs after the electrospinning process. The formed nanocomposite can be used in wide variety of applications including nanoelectronics and gas adsorption.
\end{abstract}

\section{Introduction}

Carbon fibers are of great technological and industrial interest because of their promising characteristics such as high strength to weight ratio, excellent chemical resistance, and superior electrical and thermal conductivity $[1,2]$. They have been used to develop high performance fiberreinforced composites, which are highly desirable in automotive, aerospace, and sport industries. Carbon nanofibers (CNFs) have high specific surface area, which resulted from pore-creating surface modification, which makes them suitable for gas adsorption and water treatment applications $[3,4]$. Despite the extended research in carbon composites nanofibers, the present work introduces a new nanocomposite to generate carbon nanofibers embedded into polyvinyl alcohol (PVA) electrospun nanofibers. This novel research aims to offer a cheap and biodegradable host for already synthesized carbon nanofibers instead of the deposition techniques such as sputtering or physical vapor deposition, which can impact the carbon nanofibers structure. The formed nanofibers should be highly conductive based on the superior conductivity of carbon nanofibers. The formed nanocomposites can be helpful in electronic devices, batteries, and super capacitors [5]. Electrospinning technique is selected as the fabrication method for the proposed nanocomposite because of the simplicity of operation, the feasibility to embed CNFs in the resulting PVA nanofibers, and the potential for scaleup to manufacture large volumes $[6,7]$. In this process, high electric field strength is applied between a metallic needle and a metallic target. The strength of the electric field forces the polymer droplet at the needle tip to stretch and to deposit into fibers at the surface of the target [8-11]. The resulting webs can be feasible for many applications as in nanoagriculture, tissue engineering, and biomedical applications [12-16]. 


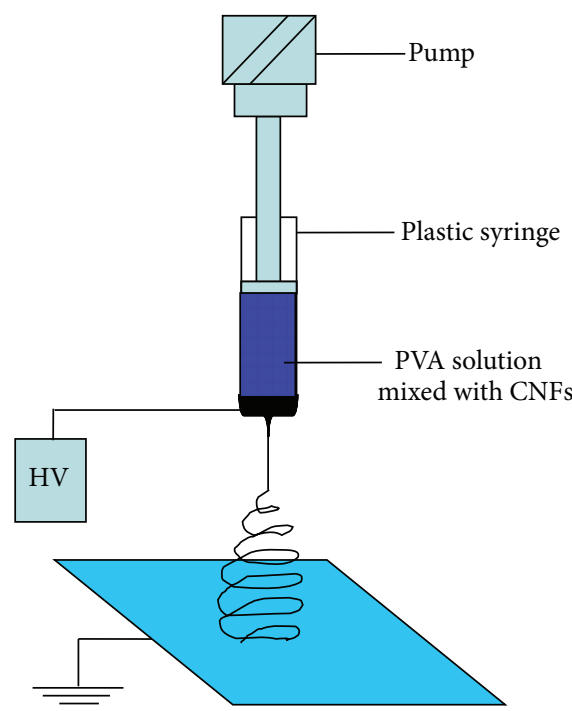

FIGURE 1: Schematic illustration of the setup of the electrospinning device.

\section{Experimental Work}

2.1. Materials. Carbon nanofibers from Sigma Aldrich, composed of graphitized (iron-free) conical platelets, have been used. In addition, polyvinyl alcohol (PVA) is used as the host material of electrospun nanofibers, with $88 \%$ degree of hydrolysis from Dupont, Taipei, Taiwan. Based on the authors' experimental work, PVA is dissolved in deionized water at concentrations $13 \mathrm{wt} \%$ for best electrospun nanofibers. In more detail, the chosen concentration is found to be optimal for producing electrospun mats with minimal visual defects such as pinholes or wet fleeces. Carbon nanofibers with different weight ratios (1 and $2 \mathrm{wt} \%$ ) are added to the PVA solution and stirred at room temperature for two hours to form a homogeneous gel.

2.2. Electrospinning. The electrospinning setup, as shown in Figure 1, consists of a $10 \mathrm{~mL}$ plastic syringe with 18-gauge metallic needle, a single polarity, and variable high voltage ( 0 $25 \mathrm{kV}$ ) power supply that is connected to the metallic target where the nanofibers can be formed. The distance between the needle tip and the target is held constant at $15 \mathrm{~cm}$. The flow rate of the polymer solution, containing PVA/CNFs, supplied to the syringe is fixed at $0.7 \mathrm{~mL} / \mathrm{hr}$. The samples are collected on a flat metallic surface covered with aluminum foil. Each deposition of electrospun fibers is run for a total of 30 minutes with the result that a nonwoven fiber web coats the target.

2.3. Characterization Methods. The mean diameter of the electrospun nanofibers is measured by FEI Quanta 200 Environmental Scanning Electron Microscope (ESEM); $5 \times$ $5 \mathrm{~mm}$ sections of nanofiber web were mounted on SEM holder. The conductivity of the electrospun nanofibers has been measured through 4-point probe station, in which Keithley 2400 voltameter measures the $I-V$ characteristics of the formed nanocomposite. Therefore, circular nanofiber mat sections were mounted on the SEM aluminum holder and fixed on a glass slide. SEM images of the webs' thickness, required for conductivity measurement, and the nanofibers are captured by ESEM and the thickness of the web has been measured. FTIR spectrum of the electrospun nanocomposite is measured by Infrared Spectrophotometer FTIR 760 (Thermo Nicolet).

Thermogravimetric analyses (TGA) of the electrospun composites have been done to observe the influence of carbon nanofibers on the degradation process of PVA. The electrospun nanofibers have been placed in an aluminum pan and heated from room temperature to $600^{\circ} \mathrm{C}$ at nitrogen atmosphere with the heating rate of $10^{\circ} \mathrm{C} / \mathrm{min}$. Differential Scanning Calorimetry (DSC) has been used to analyze the degree of crystallization using Perkin Elmer DSC 7.

\section{Results and Discussions}

Figures 2(a) and 2(b) show SEM images of both the pure electrospun PVA nanofibers and carbon nanofibers, respectively. It can be shown that the average diameter of the PVA electrospun nanofibers is $225 \mathrm{~nm}$, while the average diameter of the carbon nanofibers is $130 \mathrm{~nm}$.

Figures 3(a) and 3(b) show PVA nanofibers mixed with carbon nanofibers by 1 and $2 \%$, respectively. SEM graphs in Figure 3 show that the produced nanofibers are homogeneous and there was no observable separation between PVA nanofibers and carbon nanofibers. The nanofiber diameters of the produced PVA/CNFs composite are approximately of the same ranges of the individual nanofibers shown in Figure 2. Furthermore, carbon nanofibers are hypothesized to be stretched in the formed nanocomposite from our observation of Figure 3(b) compared to CNFs original state as shown in Figure 2(b). That may be explained due to the high electric potential in the electrospinning experiment, which helps the carbon nanofibers to be stretched.

Figure 4 shows FTIR analysis of the formed nanocomposite of CNFs hosted by PVA electrospun nanofibers. It can be observed that the formed peaks belong to the PVA only with no more bonds formed with CNFs. The observed pattern is the same compared to pure PVA nanofibers and PVA with CNFs at different weight ratios of CNFs [17].

The electrical conductivity has been increased with increasing the carbon nanofibers in the PVA nanofibers. The electrical conductivity has been increased from $1.01 \times$ $10^{-5} \mathrm{~S} / \mathrm{m}$ (for pure PVA) to $2.91 \times 10^{-5} \mathrm{~S} / \mathrm{m}$ and $16.36 \times$ $10^{-5} \mathrm{~S} / \mathrm{m}$ (for PVA nanofibers with $1 \mathrm{wt} \%$ and $2 \mathrm{wt} \%$ carbon fibers' content, resp.). The increase of the electrical conductivity of PVA/carbon fiber mat is due to the high conductivity of carbon nanofibers [18].

Figure 5 shows the TGA analysis show of pure PVA and PVA doped with CNFs. The degradation of PVA which occurs at lower temperatures by addition of carbon fibers could be observed. The onset of the weight loss temperature was reduced from $300^{\circ} \mathrm{C}$ to $279^{\circ} \mathrm{C}$ (for PVA nanofibers and $2 \mathrm{wt} \%$ carbon fibers' content, resp.). The peak of first derivative of weight loss in Figure 6 is moved from $325^{\circ} \mathrm{C}$ to $318^{\circ} \mathrm{C}$. The shifting of both the onset of weight loss and the first derivative 


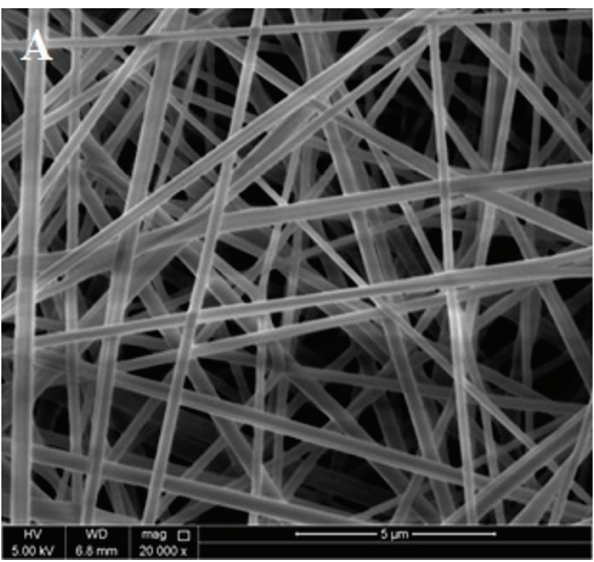

(a)

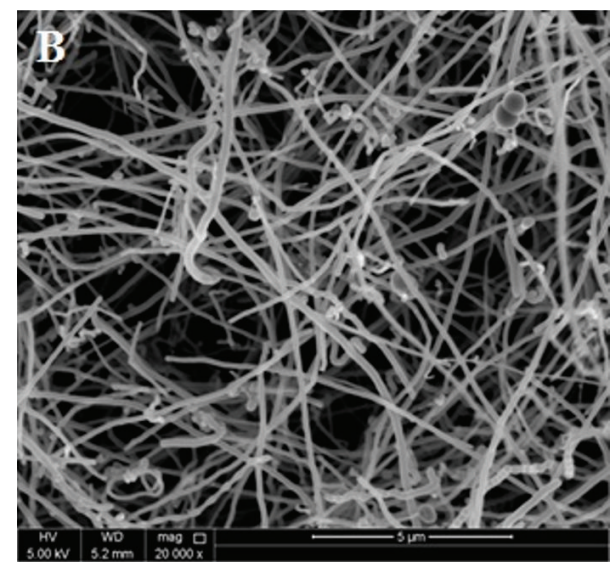

(b)

Figure 2: SEM images of (a) PVA nanofibers and (b) CNFs.

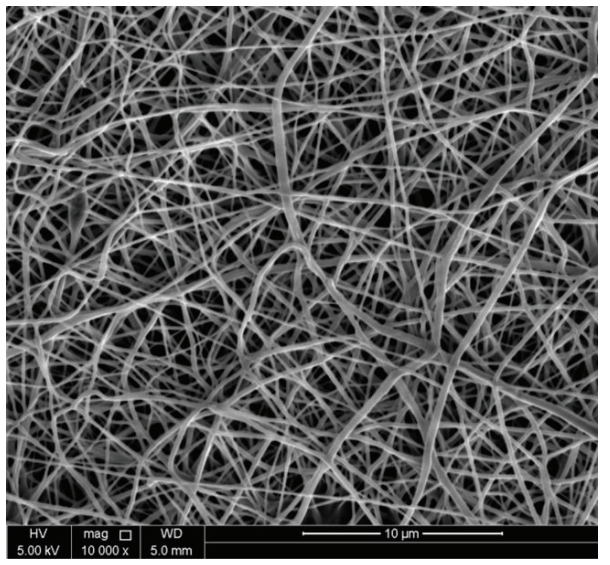

(a)

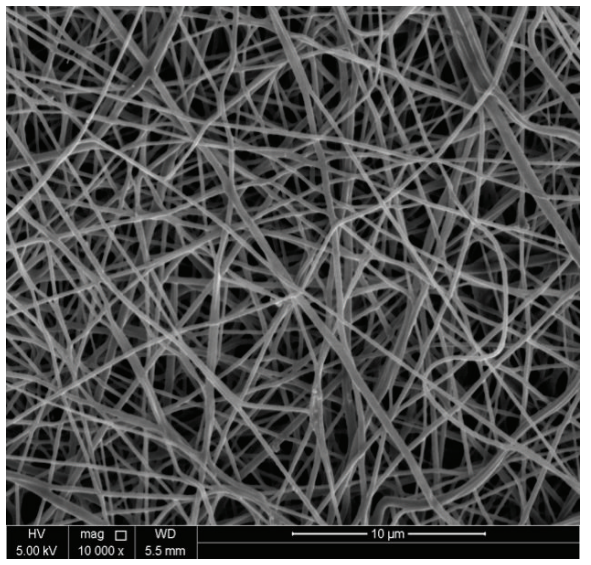

(b)

FIgURE 3: SEM images of electrospun nanofibers composed of PVA with (a) $1 \%$ wt CNFs and (b) $2 \%$ wt CNFs.

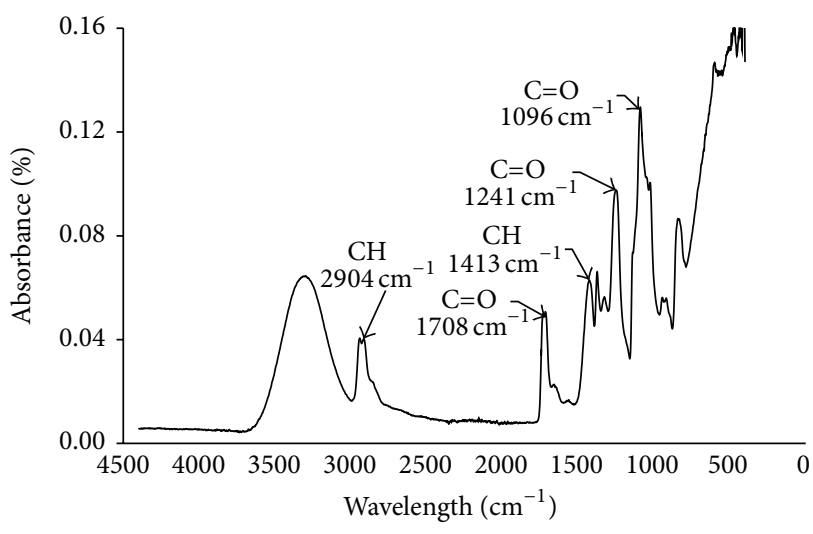

(a)

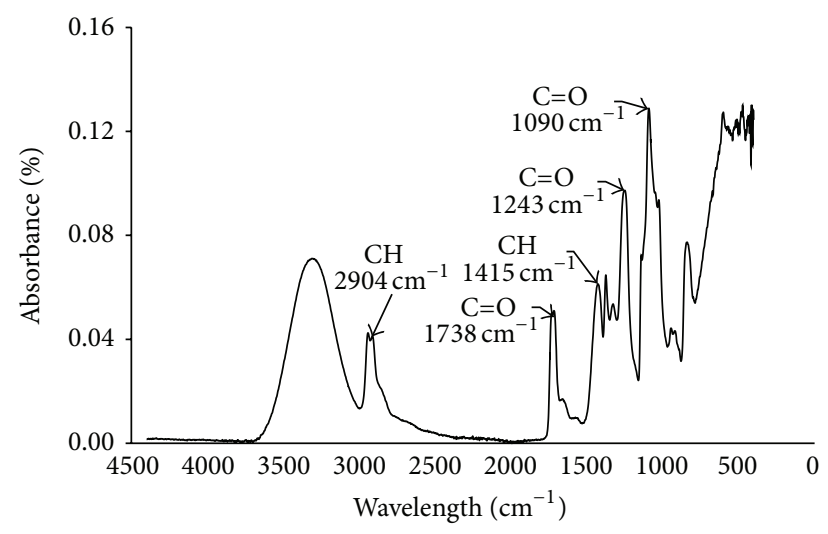

(b)

FIGURE 4: FTIR of the electrospun PVA nanofibers (a) and PVA nanofibers contain CNFs (b). 


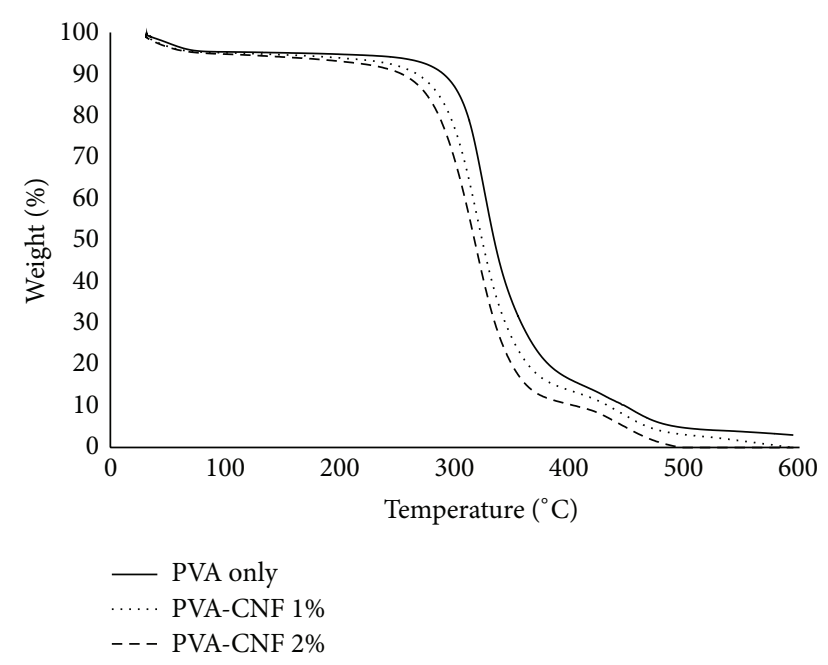

FIGURE 5: Weight loss versus temperature measured by TGA of PVA nanofibers: PVA: CNFs $1 \%$ wt nanofibers and PVA: CNFs $2 \% \mathrm{wt}$ nanofibers.

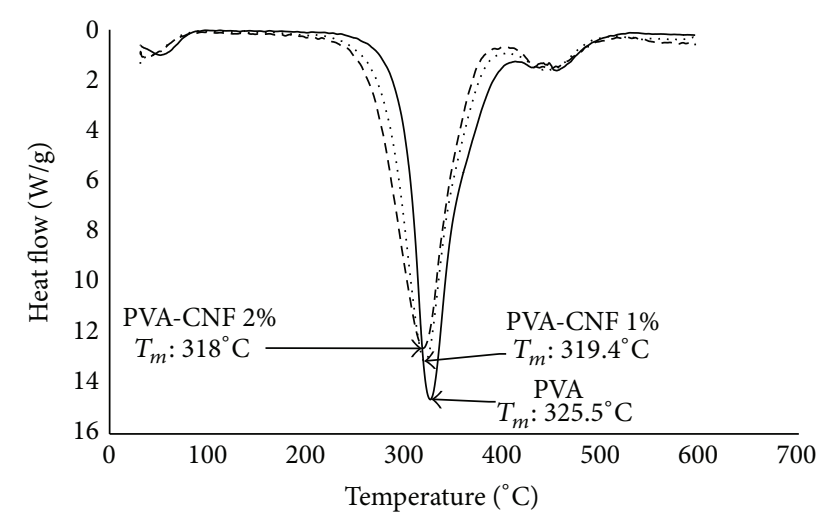

FIGURE 6: First derivative of weight loss versus temperature measured by TGA of PVA nanofibers: PVA: CNFs $1 \%$ wt nanofibers and PVA: CNFs 2\% wt nanofibers.

weight loss is due to accelerating of thermal degradation of PVA due to distributing of thermal energy within PVA matrix through thermal conductivity of carbon fibers [19].

Regarding the DSC analysis of the corresponding electrospun fibers, the melting point of PVA is shifted arbitrary from $178,186.5$, and $190^{\circ} \mathrm{C}$ for PVA and PVA with $1 \%$ and $2 \%$ CNFs, respectively. The shift of the melting point is attributed to the assistance of carbon nanofibers in increasing the degree of crystallization as they act as nucleating agents in the polymer [20]. By addition of $1 \mathrm{wt} \%$ of CNFs, the enthalpy is increased approximately $31 \%$ (from $59.3 \mathrm{~J} / \mathrm{g}$ to $77.6 \mathrm{~J} / \mathrm{g}$ ). Further addition of CNFs results in reduction to $51.4 \mathrm{~J} / \mathrm{g}$. The first increase in the enthalpy (which is related to the crystallinity percentage) can be attributed to the increase of crystallization process as carbon nanofibers enhance the nucleation and crystallization of PVA. Further additions of CNFs cause reduction in crystallinity percentage, which may be due to agglomeration of carbon nanofibers inside the PVA matrix, which reduce the required surfaces for nucleation and crystallization [20].

\section{Conclusions}

This paper investigates a new nanocomposite composed of both PVA and carbon nanofibers integrated in one electrospun nanofibers web. This technique shows a simple and cheap way to offer a host for CNFs without affecting them using traditional deposition techniques. Our results show that thermal and electrical conductivities of the formed nanofibers have been improved with increasing of the carbon nanofibers content. SEM images show the homogeneity of the formed PVA and carbon nanofibers in one web, with stretched CNFs after the electrospinning process. TGA results show that the degradation of PVA nanofibers is favored by addition of carbon nanofibers due to increase of thermal conductivity. DSC results show that addition of carbon nanofibers within PVA nanofibers increases the degree of crystallization of PVA around $31 \%$. The formed nanocomposite can be used in wide variety of applications including nanoelectronics and nanomedicine.

\section{Conflict of Interests}

The authors declare that they have no competing interests.

\section{Authors' Contribution}

Nader Shehata carried out the mixing of CNFs within PVA solution to obtain the optimum concentrations applicable for electrospinning process. In addition, Nader Shehata did the electrospinning process with selection of the optimum process parameters. Nabil Madi and Mariam Al-Maadeed both guided Nader Shehata in the overall work in addition to the critical revision of the paper. Ibrahim Hassounah contributed critically in the explanation of the resulted data especially the FTIR spectroscopy analysis. Abdullah Ashraf followed up the different characterizations done in Central Laboratory Unit (CLU) in Qatar University. All authors read and approved the final paper.

\section{Acknowledgments}

This work was funded by a QSTP grant (Award no. 0906). The authors would like to thank Central Laboratory Unit (CLU) in Qatar University for their assistance through the used characterization facilities. Also, the authors are grateful to the financial support of Center of Advanced Materials (CAM) in Qatar University and Virginia Tech Middle East and North Africa (VT-MENA) program in Egypt.

\section{References}

[1] J.-B. Donnet, T. K. Wang, S. Rebouillat, and J. Peng, Carbon Fibers, Taylor \& Francis, New York, NY, USA, 3rd edition, 1998.

[2] P. Morgan, Carbon Fibers and Their Composites, Taylor \& Francis, Boca Raton, Fla, USA, 2005. 
[3] N. Yusof and A. F. Ismail, "Post spinning and pyrolysis processes of polyacrylonitrile (PAN)-based carbon fiber and activated carbon fiber: a review," Journal of Analytical and Applied Pyrolysis, vol. 93, pp. 1-13, 2012.

[4] J. Wu and D. D. L. Chung, "Increasing the electromagnetic interference shielding effectiveness of carbon fiber polymermatrix composite by using activated carbon fibers," Carbon, vol. 40, no. 3, pp. 445-447, 2002.

[5] C. Liu, F. Li, L.-P. Ma, and H.-M. Cheng, "Advanced materials for energy storage," Advanced Materials, vol. 22, pp. E28-E62, 2010.

[6] L. Larrondo and R. S. J. Manley, "Electrostatic fiber spinning from polymer melts. I. Experimental observations on fiber formation and properties," Journal of Polymer Science: Polymer Physics, vol. 19, no. 6, pp. 909-920, 1981.

[7] Y. M. Shin, M. M. Hohman, M. P. Brenner, and G. C. Rutledge, "Electrospinning: a whipping fluid jet generates submicron polymer fibers," Applied Physics Letters, vol. 78, no. 8, article 1149, 2001.

[8] W. Liu, M. Graham, E. A. Evans, and D. H. Reneker, "Poly(metaphenylene isophthalamide) nanofibers: coating and post processing," Journal of Materials Research, vol. 17, no. 12, pp. 32063212, 2002.

[9] I. A. Hassounah, N. A. Shehata, G. C. Kimsawatde et al., "Studying the activity of antitubercluosis drugs inside electrospun polyvinyl alcohol, polyethylene oxide, and polycaprolacton nanofibers," Journal of Biomedical Materials Research Part A, vol. 102, no. 11, pp. 4009-4016, 2014.

[10] S. Matsumura, Y. Shimura, K. Terayama, and T. Kiyohara, "Effects of molecular weight and stereoregularity on biodegradation of poly(vinyl alcohol) by Alcaligenes faecalis," Biotechnology Letters, vol. 16, no. 11, pp. 1205-1210, 1994.

[11] T. Mori, M. Sakimoto, T. Kagi, and T. Sakai, "Isolation and characterization of a strain of Bacillus megaterium that degrades poly(vinyl alcohol)," Bioscience, Biotechnology and Biochemistry, vol. 60, no. 2, pp. 330-332, 1996.

[12] K. Nakamiya and S. K. Toshihiko, "Degradation of synthetic water-soluble hydroquinone peroxidase," Journal of Fermentation and Bioengineering, vol. 84, pp. 213-218, 1997.

[13] R. Marchal, E. Nicolau, J.-P. Ballaguet, and F. Bertoncini, "Biodegradability of polyethylene glycol 400 by complex microfloras," International Biodeterioration and Biodegradation, vol. 62, no. 4, pp. 384-390, 2008.

[14] A. Zgoła-Grześkowiak, T. Grześkowiak, J. Zembrzuska, and Z. Łukaszewski, "Comparison of biodegradation of poly(ethylene glycol)s and poly(propylene glycol)s," Chemosphere, vol. 64, no. 5, pp. 803-809, 2006.

[15] J. Tao, Effects of molecular weight and solution concentration on electrospinning of PVA [M.S. thesis], Worcester Polytechnic Institut, 2003.

[16] S. Matsumura, H. Kurita, and H. Shimokobe, "Anaerobic biodegradability of polyvinyl alcohol," Biotechnology Letters, vol. 15, no. 7, pp. 749-754, 1993.

[17] I. Hassounah, N. Shehata, A. Hudson, B. Orler, and K. Meehan, "Characteristics and 3D formation of PVA and PEO electrospun nanofibers with embedded urea," Journal of Applied Polymer Science, vol. 131, no. 3, Article ID 39840, 2014.

[18] L. Zhang, A. Aboagye, A. Kelkar, C. Lai, and H. Fong, "A review: carbon nanofibers from electrospun polyacrylonitrile and their applications," Journal of Materials Science, vol. 49, no. 2, pp. 463480, 2014.
[19] N. Diouri and M. Baitoul, "Effect of carbon nanotubes dispersion on morphology, internal structure and thermal stability of electrospun poly(vinyl alcohol)/carbon nanotubes nanofibers," Optical and Quantum Electronics, vol. 46, no. 1, pp. 259-269, 2014.

[20] M. A. AlMaadeed, R. Kahraman, P. Noorunnisa Khanam, and N. Madi, "Date palm wood flour/glass fibre reinforced hybrid composites of recycled polypropylene: mechanical and thermal properties," Materials and Design, vol. 42, pp. 289-294, 2012. 

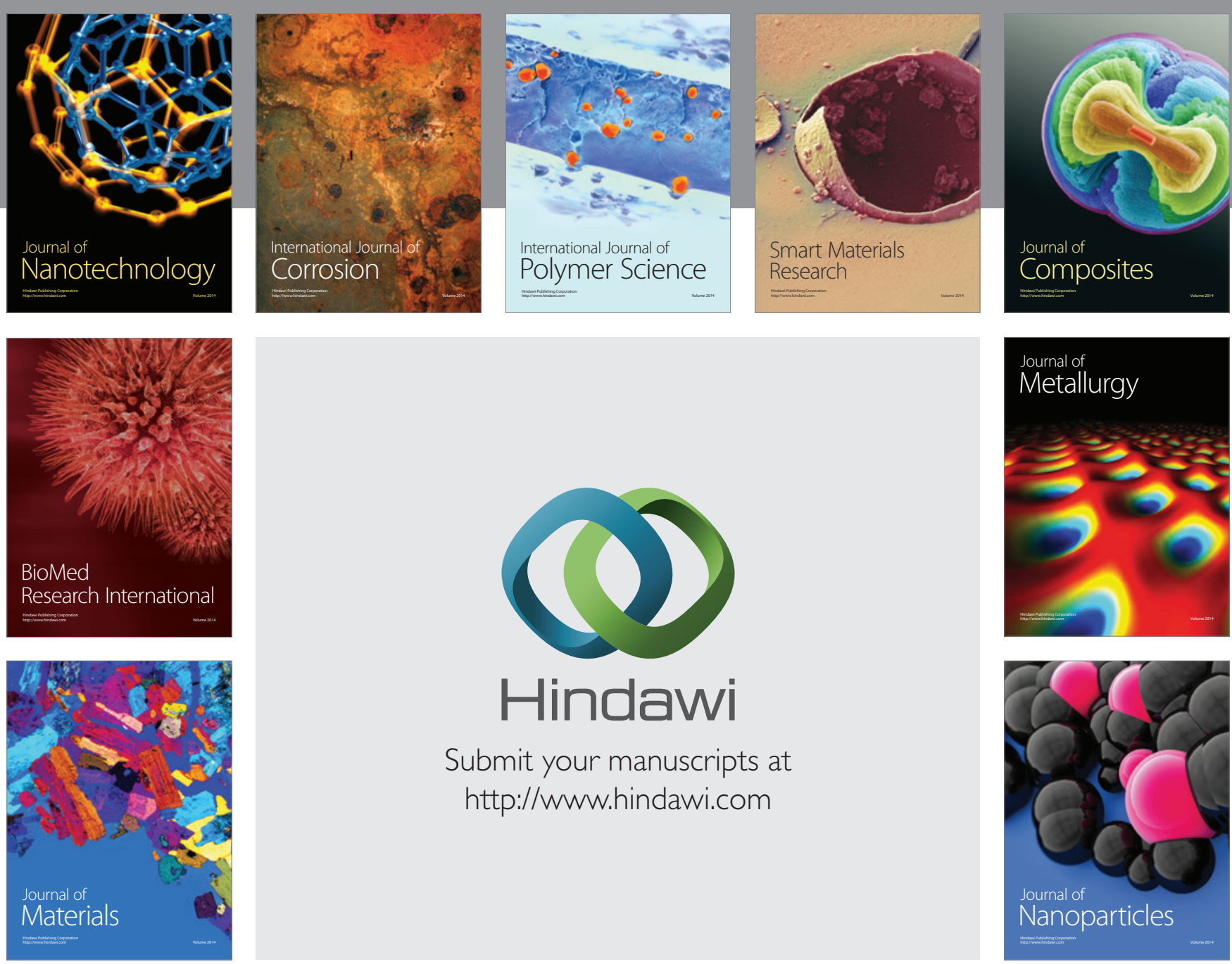

Submit your manuscripts at http://www.hindawi.com
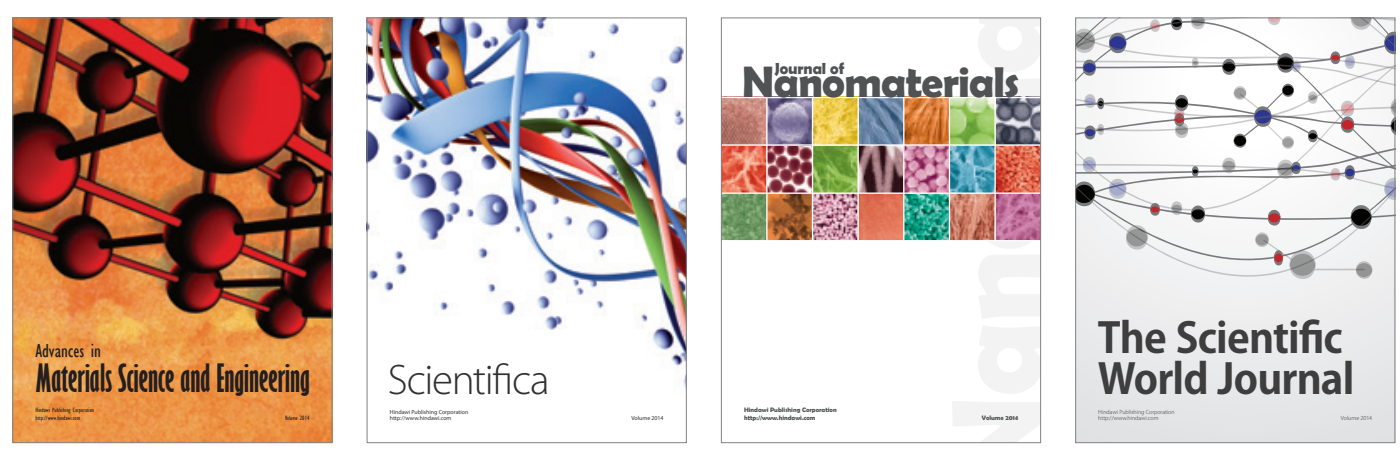

\section{The Scientific World Journal}
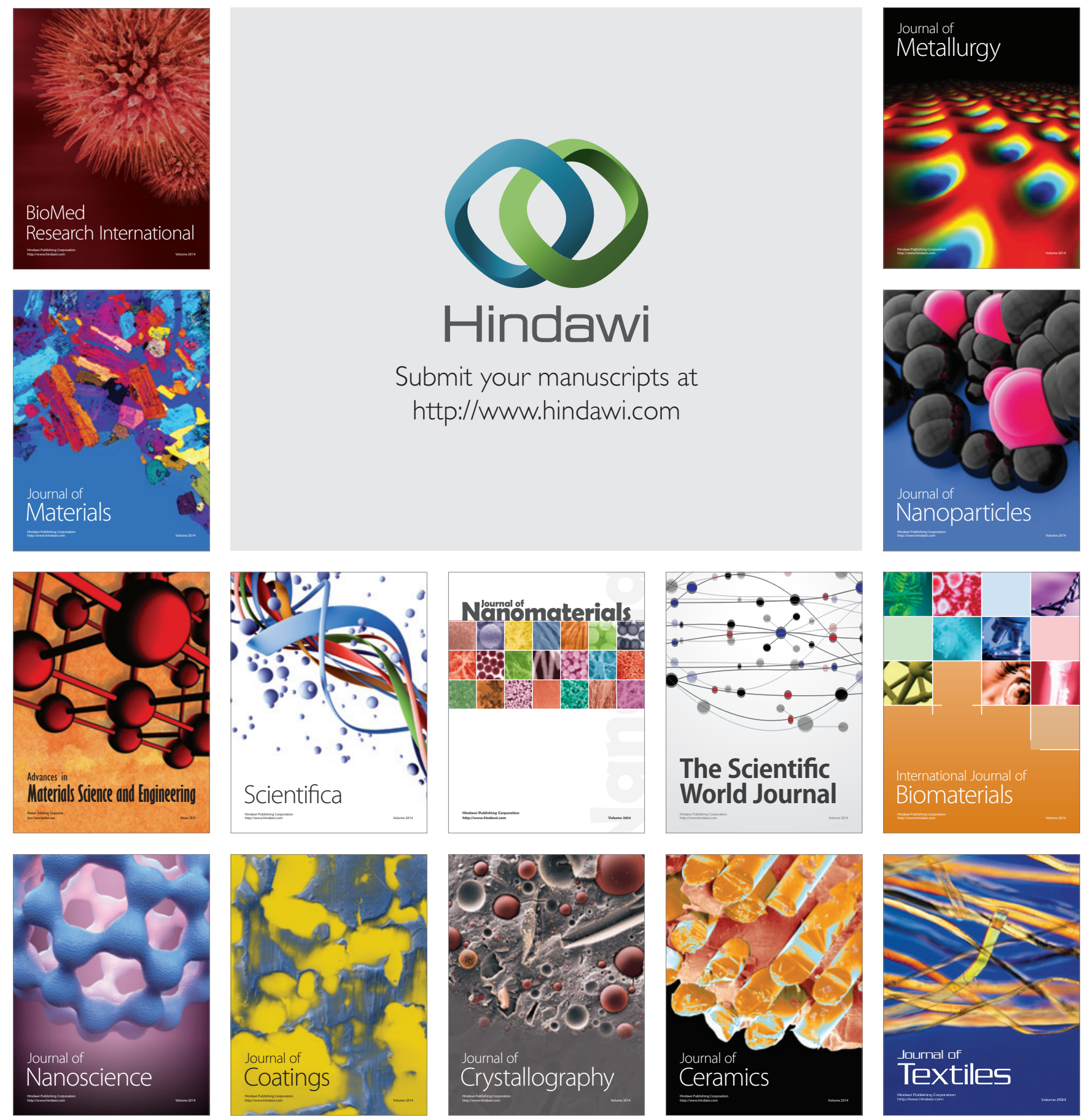